\title{
COLLAGENOUS REPEAT-CONTAINING SEQUENCE OF 26 kDa PROTEIN - A NEWLY DISCOVERED ADIPOKINE - SENSU LATO - A MINIREVIEW
}

\author{
Marek Svestak ${ }^{\mathrm{a}, \mathrm{c}}$, Lenka Sporovaa, ${ }^{\mathrm{a}, \mathrm{c}}$, Pavel Hejduk ${ }^{\mathrm{b}, \mathrm{c}}$, Borek Lacnak ${ }^{\mathrm{d}}$, David Stejskala,c* \\ ${ }^{a}$ Department of Laboratory Medicine, Central Moravian District Hospital, Prostejov, Czech Republic \\ ${ }^{b}$ Department of Nephrology, Central Moravian District Hospital, Prostejov \\ c Department of Medical Chemistry and Biochemistry, Faculty of Medicine and Dentistry, Palacky University Olomouc, \\ Czech Republic \\ ${ }^{d}$ Department of Internal Medicine, Vsetin, Czech Republic \\ E-mail: david.stejskal@nemsne.cz
}

Recived: September 1, 2009; Accepted with revision: August 5, 2010

Key words: CORS-26/Cartducin/Adipokines/Adipose tissue/Metabolic syndrome/Osteosarcoma/Chondrogenesis

Background. Increasing evidence from numerous research studies in internal medicine shows that adipocytes and adipokines are involved in primary inflammatory processes and disease. CORS-26 (collagenous repeat- containing sequence of $26 \mathrm{kDa}$ protein) is a newly discovered adipokine of the C1q/TNF molecular superfamily C1q/TNF-related protein-3 (CTRP-3) secreted, inter alia in murine monocytes and adipocytes and in human adipocytes. Reported recently as a gene product of adipocyte differentiation, it shares structural similarity with the adipocyte, adiponectin. CORS-26 is much less known than other adipocytes such as leptin and resistin. Knowledge of its various functions has clinical and therapeutic implications especially in relation to obesity and the metabolic syndrome.

Aims. This review aims to provide current knowledge of this adipokine.

Methods. Review; sources were scientific biomedical databases Medline/PubMed, BioMedCentral, Google Scholar, Ovid, ProQuest from to 1998 to 2009.

Conclusion. CORS-26 is an adipokine that regulates the secretion of other adipokines. Its effects on adipokine secretion are most probably independent of PPAR- $\gamma$. As CORS-26 up-regulates adiponectin secretion, it may be involved in metabolic and immunologic pathways. The effect of recombinant CORS-26 on insulin signaling in the presence of the metabolic syndrome needs to be investigated to further evaluate the physiological and pathophysiological role of this protein.

\section{INTRODUCTION}

Human and murine CORS-26 (collagenous repeatcontaining sequence of $26 \mathrm{kDa}$ protein, also known as Cartonectin, Cartducin, CTRP3 (C1q/TNF-related protein 3) and CIATNF3 (Complement C1q tumor necrosis factor-related protein 3 ) is a 246 amino acid sequence protein with secretory signal peptide, collagenous region and globular C-terminal domain. It circulates in a high molecular weight form in plasma and it is converted to a lower molecular weight form by the denaturing conditions of the systemic circulation ${ }^{1}$. It is an assumed paralog of adiponectin. Adiponectin comprises 244 amino acids containing a short noncollagenous $\mathrm{N}$-terminal domain followed by a collagen-like sequence with 22 perfect Gly-X-Pro motifs ${ }^{1}$. This collagen domain is followed by a C-terminal globular domain that forms the so-called "bouquet of flowers" structure of the oligomerization complex with striking homology to the complement component C1q. The open reading frame of CORS-26 predicts a polypeptide sequence of 246 amino acids corresponding to a calculated molecular mass of $26 \mathrm{kDa}$. The presence of a signal peptide of 22 amino acid residues with a predicted cleavage site at Cys 22 suggests that CORS-26 is a secretory protein like adiponectin ${ }^{1-3}$.
The C-terminal globular domain is homologous to complementC1q, and is thought to be a functional domain that may interact with other proteins or receptors.

The murine gene encoding this protein was cloned by Maeda et al. ${ }^{4}$ in 2001 and the approved gene symbol is C1QTNF3( ref. $^{1}$ ).

The human homolog of the mouse CORS-26 gene has also been identified and mapped to the chromosome locus $5 \mathrm{p} 13.2-13.1$ which is close to the locus of the growth hormone receptor (GHR) gene $(5 \mathrm{p} 13.1-12)^{2,3,5}$.

\section{CORS-26 EXPRESSION}

CORS-26 mRNA expression has been found in hormonally differentiated $3 \mathrm{~T} 3-\mathrm{L} 1 \mathrm{cells}^{2,3}$. Whereas adiponectin is exclusively expressed by adipocytes, CORS-26 mRNA is also detected in cartilage, kidney, colon, small intestine, placenta, lung, spleen, fibroblasts, human monocytic and dendritic cells ${ }^{5,6}$. In addition, strong CORS-26 mRNA expression has been found in osteosarcoma, chondroblastoma and giant cell tumors ${ }^{2,3,6}$. 


\section{CORS-26 EFFECTS}

\section{CORS-26 in the regulation of osteosarcoma cells}

Because the murine gene on chromosome 15A2 maps to a linkage locus for two animal models of arthritis (proteoglycaninduced arthritis and murine 1pr-mutation/lpr arthritis) and because the human gene locus on chromosome 5 p 13 is affected by chromosomal imbalances in the presence of osteosarcoma, a putative function of CORS-26 in the development of mesenchymal tissue can be assumed. In this context, it is important to note that human CORS-26 mRNA is up-regulated in osteosarcoma cells. These data also indicate a role for CORS-26 in the context of mesenchymal tissue development and in the pathogenesis of bone and skeletal diseases ${ }^{6}$. Akiyama et al. ${ }^{7}$ have reported that stimulation of osteosarcoma cells by CORS-26 promotes tumor cell growth but not migration in vitro. Osteosarcoma tumor growth is associated with activation of the ERK1/2 signaling pathway?.

\section{CORS-26 effect on chondrogenesis and cartilage development}

CORS-26 has been shown to be a novel growth factor that plays an important role in regulating both chondrogenesis and cartilage development (CORS-26 gene is predominantly expressed in growth plate cartilage $)^{5,8}$. Skeletal development requires condensation of multipotential mesenchymal cells in order to differentiate into various cell types. One such process is the formation of chondrocytes from undifferentiated mesenchymal cells. Chondrocytes then continue to grow by proliferation, maturation, and matrix deposition under the regulation of several hormones and growth factors.

Although the CORS-26 gene is known to be expressed predominantly in growth plate cartilage, localization of its transcripts in this tissue is still unclear. CORS-26 transcripts were localized in proliferative zones but not in prehypertrophic or hypertrophic zones of the epiphyseal growth plate cartilage . $^{2}$

No specific signal was detected in bone tissue and no CORS-26 mRNA expression was observed in fibroblastlike or osteoblast-like cells.

These findings indicate that only proliferating chondrocytes in growth plate cartilage express CORS-26 suggesting that CORS-26 could be a useful marker for chondrogenic precursors 5 .

Recombinant CORS-26 has been shown to be a growth factor that promotes the proliferation of chondrogenic progenitor N1511 cells, and chondrocytic HCS-2/8 cells in a dose-dependent manner ${ }^{5}$.

\section{CORS-26 in the pathophysiology of the metabolic syndrome (MS)}

The location of the murine gene is very close to the microsatellite marker D15Mit225. Some authors describe an association between fasting glucose levels with the centromeric region on chromosome 15 near D15Mit225, with a maximum logarithmic odds ratio score in the region containing the CORS-26 gene 9 .
An association of serum leptin levels with genomic loci in French sibpairs suffering from obesity has been described and a link of leptin with the region between microsatellite markers D5S477 and D5S426 containing the CORS-26 gene has been confirmed ${ }^{10}$. A link for the association of diabetes and BMI with chromosomal loci in Pima Indians has been identified (between microsatellite markers D5S1470 and D5S426 containing the CORS-26 gene $)^{11}$.

Because CORS-26 is specifically induced during late adipocyte differentiation and because it stimulates the secretion of adiponectin and resistin, a putative role in the context of insulin resistance, type 2 diabetes and the MS can be suspected ${ }^{1}$.

Adiponectin is a highly abundant circulating adipokine secreted by differentiated adipocytes. It is decreased in obese and diabetic humans and animals. It has has antiinflammatory and anti-diabetic effects which have been demonstrated in numerous in vitro studies and animal models. It forms trimers, hexamers and higher molecular weight (HMW) complexes, and these isoforms have been detected in sera and conditioned media of differentiated 3T3-L1 adipocytes. Recent studies indicate that the HMW adiponectin, like CORS-26 is the active form of the protein ${ }^{12}$. Total circulating adiponectin is significantly decreased in obese and diabetics patients and individuals at high risk of cardiovascular disease. In contrast to leptin, adiponectin and CORS-26 like omentin are not influenced by oral glucose load. It seems that systemic adiponectin and CORS-26 are not influenced by acute hyperglycemia or hyperinsulinemia ${ }^{12,13}$.

CORS-26 used in concentrations ranging from 10 to $50 \mu \mathrm{g} / 1$ was not found to stimulate leptin, interleukin-6 (IL-6) or adiponectin secretion from human adipocytes. However, high doses of CORS-26 increased adiponectin and leptin secretion in murine adipocytes but did not modulate PPAR- $\gamma$ expression ${ }^{14}$.

Since the receptor for CORS-26 has not yet been identified, it is possible that the specific receptor might be expressed only in murine and not human adipocytes. As adiponectin is a more anti-inflammatory protein and resistin is a more pro-inflammatory protein, CORS-26 seems to regulate both pro- and anti-inflammatory functions of adipose tissue (Fig. 1).

It is important to emphasize that adipose tissue becomes infiltrated by significant amounts of monocytes in the context of obesity. These monocytes are attracted by adipose tissue-derived chemo-attractants such as monocyte chemo-attractant protein-1, and these secrete proinflammatory molecules that influence local adipocyte function and insulin sensitivity. In this regard, it has been recently demonstrated that CORS-26 is produced by monocytic cells and exerts potent anti-inflammatory properties by reducing IL- 6 and TNF- $\alpha$, most probably through suppression of NF-kB signaling. In this manner, adipocyte- and monocyte-derived CORS-26 might act as a new immunomodifying molecule within adipose tissue. Regarding insulin sensitivity, CORS-26 induces both the anti-diabetic adiponectin and the pro-diabetic resistin. 


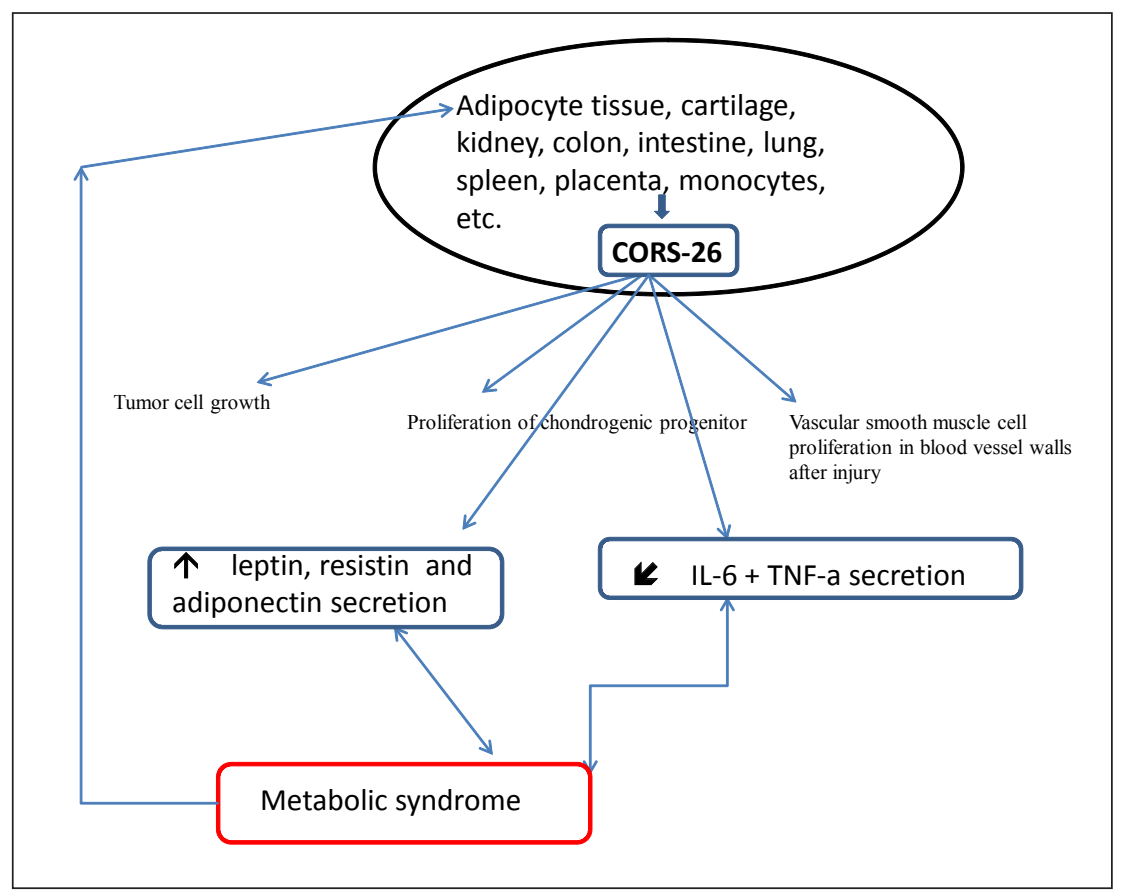

Fig. 1. Effects of CORS-26.

Therefore, CORS-26 might also exert profound effects on local insulin sensitivity within adipose tissue ${ }^{14}$.

CORS-26 and the pathophysiology of neointimal hyperplasia and restenosis following angioplasty

CORS-26 positively regulates two key steps in angiogenesis, namely, proliferation and migration of endothelial cells via distinct MAPK signaling pathways ${ }^{8}$. Recently a novel biological role for CORS-26 in promoting vascular smooth muscle cell proliferation in blood vessel walls after injury, has been proposed (Fig. 1) (ref. ${ }^{15}$ ). CORS-26 expression is up-regulated transiently. These results suggest that CORS-26 may play an important role in the pathophysiology of neointimal hyperplasia and restenosis following angioplasty ${ }^{8}$.

\section{CORS-26 effect on cytokine values}

CORS-26 reduces IL-6 and TNF-a secretion but does not increase IL-10 in primary monocytes cells (Fig 1). Suppression of NF-kB signalling may explain the anti-inflammatory actions of CORS-26. These data demonstrate that CORS-26 forms trimers, exerts anti-inflammatory effects and that it is expressed in monocytic cells. Therefore CORS-26 may provide a new target for pharmacological drugs for use in inflammatory diseases like the metabolic syndrome ${ }^{5}$.

\section{METHOD OF CORS-26 MEASUREMENT}

\section{CORS-26 gene expression}

CORS-26 expression was assessed using hybridization (a method with lower sensitivity) and RT-PCR techniques where CORS-26 mRNA was easily amplified from total RNA isolated from cells (monocytes) ${ }^{5,6}$.

\section{CORS-26 protein concentration}

Qualitative analysis of CORS-26 protein was made by imunoblot (impractical method) ${ }^{13}$.

Recently immunoassay for routine quantitative determination of human CORS-26 in serum/plasma was established and evaluated ${ }^{13}$.

\section{CONCLUSIONS}

CORS-26 is a new adipokine that differentially regulates the secretion of other adipokines from adipocytes. Its effects on adipokine secretion are most probably independent of PPAR- $\gamma$ mediated promoter activation. Since CORS-26 up-regulates adiponectin secretion, it might be involved in metabolic and immunologic pathways.

The effect of recombinant CORS-26 on insulin signaling in the presence of the metabolic syndrome needs to be investigated to further evaluate the physiological and pathophysiological role of this protein.

\section{ACKNOWLEDGEMENT}

Financial support from the Czech Ministry of Education, Youth and Sports (Grant No. MSM6198959216) is gratefully acknowledged.

\section{REFERENCES}

1. Schäffer A, Weigert J, Neumeier M, Schölmerich J, Buechler C. Regulation and Function of Collagenous Repeat Containing Sequence of 26-kDa Protein Gene Product "Cartonectin". Obesity 2007; 15:303-313. 
2. Schäffler A, Ehling A, Neumann E, Herfarth H, Paul G, Tarner I, Gay S, Buechler C, Schölmerich J, Müller-Ladner U. Role of specificity protein-1, PPARgamma, and pituitary protein transcription factor-1 in transcriptional regulation of the murine CORS-26 promoter. Biochim Biophys Acta 2004; 1678:150-156.

3. Schäffler A, Ehling A, Neumann E, Herfarth H, Tarner I, Gay S, Schölmerich J, Müller-Ladner U. Genomic organization, chromosomal localization and adipocytic expression of the murine gene for CORS-26 (collagenous repeat-containing sequence of $26 \mathrm{kDa}$ protein). Biochim Biophys Acta 2003; 1628:64-70.

4. Maeda $\mathrm{T}$ et al. Molecular cloning and characterization of a novel gene, CORS26, encoding a putative secretory protein and its possible involvement in skeletal development. J Biol Chem 2001; 276: 3628-3634.

5. Maeda T, Jikko A, Abe M, et. al. Cartducin, a Paralog Acrp30/ Adiponectin, Is Induced During Chondrogenic Differentiation and Promotes Proliferation of Chondrogenic Precursors and Chondrocytes. J Cell Physiol 2006; 206:537-544.

6. Weigert J, Neumeier M, Schaffler A, et.al. The adiponectin paralog CORS-26 has anti-inflammatory properties and is produced by human monocytic cells, FEBS Letters 2005; 579:5565-5570.

7. Akiyama H, Furukawa S, Wakisaka S, Maeda T. Elevated expression of CTRP3/cartducin contributes to promotion of osteosarcoma cell proliferation. Oncol Rep 2009; 21:1477-1481.

8. Akiyama H, Furukawa S, Wakisaka S, Maeda T. CTRP3/cartducin promotes proliferation and migration of endothelial cells. Mol Cell Biochem 2007; 304:243-248.
9. Shike T, Hirose S, Kobayashi M, Funabiki K, Shirai T, Tomino Y. Susceptibility and negative epistatic loci contributing to type 2 diabetes and related phenotypes in a KK/Ta mouse model. Diabetes 2001; 50:1943-1948.

10. Hager J, Dina C, Francke S, et al. A genome-wide scan for human obesity genes reveals a major susceptibility locus on chromosome 10. Nat Genet 1998; 20:304-308.

11. Lindsay RS, Kobes S, Knowler WC, Bennett PH, Hanson RL. Genome-wide linkage analysis assessing parent-of-origin effects in the inheritance of type 2 diabetes and BMI in Pima Indians. Diabetes 2001; 50:2850-2857.

12. Aso $\mathrm{Y}$, Yamamoto R, Wakabayashi $\mathrm{S}$ et al. Comparison of Serum High-Molecular Weight (HMW) Adiponectin With Total Adiponectin Concentrations in Type 2 Diabetic Patients With Coronary Artery Disease Using a Novel Enzyme-Linked Immunosorbent Assay to Detect HMW Adiponectin. Diabetes 2006; 55:1954-1960.

13. Wurm S, Neumeier M, Weigert J, Schäffler A, Buechler Ch. Plasma levels of leptin, omentin, collagenous repeat-containing sequence of 26-kDa protein (CORS-26) and adiponectin before and after oral glucose uptake in slim adults. Cardiovasc Diabetol 2007; 6:7.

14. Wölfing B, Buechler $\mathrm{CH}$, Weigert J, et.al. Effects of the New C1q/ TNF-related protein (CTRP-3) "Cartonectin" on the Adipocytic Secretion of Adipokines. Obesity 2008; 16:1481-1486.

15. Maeda T, Wakisaka S. CTRP3/cartducin is induced by transforming growth factor-beta 1 and promotes vascular smooth muscle cell proliferation. Cell Biol Int 2010; 34:261-266. 